\title{
¿Qué dicen los números de la evolución temporal de la enfermedad de Chagas?
}

\author{
Mauricio Canals, Christian González, Lucia Canals, Andrea Canals, Dante Cáceres, \\ Sergio Alvarado, Pedro E. Cattan, Miguel Saavedra, Inés Zulantay y Werner Apt
}

Universidad de Chile. Facultad de Medicina. Escuela de Salud

Pública, Programa de Salud Ambiental (MC, AC, DC, SA).

Departamento de Medicina (MC) Instituto de Ciencias BioMédicas, Laboratorio de Parasitología (MS IZ, WA)

Facultad de Ciencias Veterinarias y Pecuarias. Departamento de Ciencias Biológicas Animales (PEC)

Clínica Santa María.

Dirección Académica (AC). Instituto de Salud Pública.

Laboratorio de Entomología Médica (CG).

Universidad Metropolitana de

Ciencias de la Educación.

Departamento de Entomología

Trabajo financiado por Fondecyt 1150514

Recibido: 17 de octubre de 2016 Aceptado: 21 de marzo de 2017

Correspondencia a: Mauricio Canals Lambarri mcanals@uchile.cl

\section{What do the numbers tell us about the temporal evolution of Chagas' disease?}

Chagas disease remains highly prevalent in Chile, especially between the regions of Arica and Parinacota, and Coquimbo. Since 1999 it is considered that in Chile the vector transmission was interrupted. Under this premise, the epidemiological dynamics should be changing. We analyzed the evolution of the prevalence of Chagas' disease analyzing 64,995 xenodiagnosis performed in the laboratory of Parasitology of the Faculty of Medicine of the University of Chile between 1949 and 2014. The evolution of the mortalities and incidences from the databases of the Ministry of Health in the periods in which it was analyzed. The rates of domiciliary infestation and the number of vector insects sent to the Public Health Institute and its trypano-triatomine indices were also analyzed. The prevalence of Chagas' disease in inhabitants of risk areas remained stable in this period as well as mortality. The incidence rate shows a progressive increase with a tendency towards stabilization. A significant decrease in sampling effort was found, declining by two orders of magnitude, especially since 2000 . The progressive increase in morbidity had no clear relation to the interruption of the vector chain nor to the greater diagnostic effort occurred in 2009, since it was evident from before. While home infestation declines, reports of intrusion of solitary individuals and wild foci of $T$. infestans have increased. Trypano-triatomine indices were maintained with high values in all vector species. This study shows a worrying situation, for while on the one hand the interruption of the vector transmission and improvement in the research systems is emphasized, the concern for this disease seems to be decreasing with less diagnostic efforts and lower education at the higher level, and by the other hand the numbers show that the problem if it is not increasing, at least maintains its careless historical magnitude.

Key words: Chagas disease, epidemiology, Chile.

Palabras clave: Enfermedad de Chagas, epidemiología, Chile.

\section{Introducción}

L a enfermedad de Chagas es una de las principales enfermedades desatendidas que afecta a las Américas y que actualmente se ha constituido en una enfermedad emergente en algunas partes de América y en Europa ${ }^{1-3}$, siendo incluso comparada con la etapa temprana de la epidemia de infección por VIH/SIDA ${ }^{4}$. La incidencia anual varía entre 28.000 y 56.000 personas, entre 10.000 y 14.000 muertes anuales ${ }^{1,4}$, afectando entre 6 y 11 millones de individuos ${ }^{5}$ con 65 a 100 millones de personas en riesgo ${ }^{2,3,6}$. El área hiperendémica en Chile se extiende entre las regiones de Arica-Parinacota- $\mathrm{XV}^{\circ}$ Región (18 30'S) y del Libertador Bernardo O'Higgins$\mathrm{VI}^{\circ}$ Región (34 $36^{\prime} \mathrm{S}$ ) con una población en riesgo de 873.415 personas $^{2}$.

La enfermedad de Chagas es producida por el protozoo Trypanosoma cruzi, transmitido en Chile por los vectores Triatoma infestans, Mepraia spinolai, Mepraia gajardoi y Mepraia parapatrica (Hemíptera, Reduviidae, Triatominae), siendo $T$. infestans el vector domiciliario ${ }^{7-18}$ y el principal responsable de la prevalencia de esta enfermedad en este país ${ }^{19-22}$. Existen además otras formas de transmisión tales como la congénita, oral, por transfusiones y accidentes, que en Chile tienen una importancia menor ${ }^{2,23-26}$. Sin embargo, Uruguay desde 1997, Chile desde 1999 y Brasil desde 2006 han sido reconocidos como países en que la cadena de transmisión por $T$. infestans ha sido interrumpida a consecuencia de eficientes campañas de erradicación del vector ${ }^{2,27-29}$.

La consecuencia de la interrupción de la transmisión por $T$. infestans debería estar produciendo un cambio desde una transmisión vectorial a una congénita, lo que tendría consecuencias en la dinámica de transmisión, el número reproductivo $\left(R_{0}\right)$ de la enfermedad, prevalencia, incidencia e índices tripano-triatominos ${ }^{29-31}$.

La última encuesta nacional de salud (ENS) reporta una prevalencia de infección por $T$. cruzi de $0,7 \%$ de la 
población, con una prevalencia de $1,5 \%$ en zona rural y de $0,6 \%$ en zona urbana ${ }^{3,32}$, y los informes ministeriales reportan una infestación domiciliaria por $T$. infestans prácticamente inexistente ${ }^{3}$, lo que contrasta fuertemente con los datos reportados en los años 80 y 90 's. Por ejemplo, entre 1937 y 1980 se reportaba una prevalencia general de $16,7 \%$ en zonas endémicas con un máximo de $43,6 \%$ en la Región de Coquimbo-IV ${ }^{\circ}$ Región ${ }^{7}$, lo que no había variado significativamente entre $1982-1985^{33}$, mientras que entre 1982-1989 ya se reportaban diferencias entre zonas rurales con prevalencias de $16,7 \%$ y zonas urbanas con prevalencias de $1,9 \%{ }^{8}$. Lo mismo ocurre con la infestación domiciliaria, en que reportes previos señalaban infestaciones entre 26,8 y 33,2\% de las viviendas en zonas endémicas entre Arica-Parinacota y la Región de O’Higgins ${ }^{3,7}$.

Esto produce la falsa impresión que la enfermedad de Chagas ya no es un problema en Chile, lo que tiene incidencia en la dedicación y esfuerzo invertido en la prevención y control de esta enfermedad e incluso en la enseñanza, sumergiéndola como una más de las enfermedades desatendidas ${ }^{4}$. Sin embargo, llaman la atención algunas cifras que revelan tendencias ascendentes en la incidencia de la enfermedad ${ }^{3}$ y el reporte cada vez más frecuente de focos silvestres del vector principal, $T$. infestans $^{34-36}$. En este panorama, es difícil formarse una impresión de la evolución temporal y el real estado de la enfermedad de Chagas en Chile.

El objetivo de este trabajo es analizar la evolución temporal de la enfermedad de Chagas en Chile, mirado desde diversas perspectivas y fuentes de información publicadas, intentando formarse una idea de la real dimensión de la enfermedad.

\section{Material y Métodos}

Se abordó el análisis desde las siguientes perspectivas:

- Evolución de la prevalencia

- Evolución de la incidencia y mortalidad

- Evolución de la infestación domiciliaria

- Evolución del reporte de vectores.

Para el análisis de la evolución de la prevalencia, la fuente de información fue el registro de todos los xenodiagnósticos realizados desde 1949 en el Laboratorio de Parasitología de la Facultad de Medicina de la Universidad de Chile. Este registro se encontraba en libros disponibles en el laboratorio, siendo la información digitalizada y almacenada en formato EXCEL. Este registro corresponde a pacientes sospechosos de enfermedad de Chagas provenientes de zonas hiperendémicas. En total fueron 64.995 estudios realizados en 65 años. Este registro fue iniciado por el Dr. Amador Neghme y consta con las contribuciones de muchos equipos que colaboraron en desarrollarlo, entre ellos los Dres. Víctor Bertín, Roberto
Gajardo-Tobar, Hugo Schenone y Werner Apt, hasta nuestros días.

Para la evolución de la incidencia, mortalidad e infestación domiciliaria se recurrió a la información publicada, disponible en la red proveniente del Departamento de Epidemiología del Ministerio de Salud ${ }^{3,37}$. La serie de incidencias se analizó desde 1987 a 2014 y la serie de mortalidades desde 1985 a 2013.

Para la evolución de las poblaciones de vectores se utilizaron los registros de triatominos y otros hemípteros recibidos en el Instituto de Salud Pública entre el 1 de enero de 2005 y julio de 2016. Desde 2005, el Laboratorio de Entomología Médica de la Sección Parasitología del Instituto de Salud Pública de Chile (ISP), colabora en las campañas de vigilancia y control de triatominos desarrolladas por el Ministerio de Salud. En este laboratorio se determinan taxonómicamente las especies colectadas en las Oficinas Provinciales de las SEREMIS de Salud. Además se determina la infección por T. cruzi. Para esto se realiza la disección y extracción de la parte terminal del tubo digestivo, el cual se somete a la separación del material genético mediante un método simple por calor, y luego a la detección de secuencias específicas del kinetoplasto de $T$. cruzi (kDNA), utilizando los partidores 121/122 mediante reacción en cadena de polimerasa (RPC) convencional, metodologías realizadas en el Laboratorio de Biología Molecular de la Sección Parasitología del ISP.

Toda la información obtenida de estas fuentes fue contrastada con información previa obtenida de la literatura científica.

El análisis estadístico consistió en análisis de regresión realizados con los software STATA 11.0 y STATISTICA 7.0. Además, en el caso de la incidencia y la mortalidad, se realizaron análisis de la covarianza para comparar las pendientes de crecimiento en las etapas pre y post corte de la cadena de transmisión ocurrida en 1999 y para comparar las etapas pre y post mejoría de los métodos diagnósticos y tamizaje ocurrido en 2009.

\section{Resultados}

La prevalencia de la enfermedad de Chagas en los pacientes referidos al laboratorio se ha mantenido constante durante los 65 años que abarcó el análisis (Pendiente $\beta$ n.s., $\mathrm{R}^{2}=0,002 ; \mathrm{F}_{1,64}=0,01, \mathrm{p}=0,92$ ) (Figura 1). La prevalencia promedio fue 9,35 $\pm 0,1 \%$; $\mathrm{IC}_{0,95}:[9,12 ; 9,57]$. El esfuerzo de muestreo estimado a través del número de exámenes realizados cada año ha presentado una caída progresiva a lo largo de los años $\left(\beta=-37,3, R^{2}=0,45 ; F_{1,64}=53,2, p<<0,001\right)$ (Figura 2) y no se correlacionó con la prevalencia estimada ( $\beta$ n.s., $\left.\mathrm{R}^{2}=0,002 ; \mathrm{F}_{1,64}=0,10, \mathrm{p}=0,75\right)$.

$\mathrm{La}$ incidencia muestra un incremento progresivo a lo largo de los años $\left(\beta=0,241, \mathrm{R}^{2}=0.63 ; \mathrm{F}_{1,25}=43,4\right.$, 
$\mathrm{p}<<0,001$ ) (Figura 3). No se encontraron diferencias en las pendientes entre el período pre-corte y post-corte de la cadena de transmisión ( $\beta=0,291$ y 0,293 , respectivamente; $\left.F_{1,23}=0,00025, p=0,99\right)$. El ascenso de la incidencia viene sucediendo desde antes de la mejora diagnóstica ocurrida en $2009\left(\beta=0,154, \mathrm{R}^{2}=0,52 ; \mathrm{F}_{1,19}=20,65\right.$, $\mathrm{p}<0,001)$. Ocurre un cambio en la pendiente $\left(\mathrm{F}_{1,23}=5,57\right.$, $\mathrm{p}=0,023)$ con estabilización de la incidencia $(\beta$ n.s.; $\left.\mathrm{F}_{1,4}=0,50, \mathrm{p}=0,51\right)$. La incidencia antes de 2009 tenía un valor promedio $2,7 \pm 1,29$ por 100 mil habitantes con un $\mathrm{IC}_{0,95}[2,15 ; 3,25]$, mientras que después de 2009 el promedio es $7,3 \pm 2,02$ por 100 mil habitantes con un $\mathrm{IC}_{0,95}[5,68 ; 8,92]$.

La mortalidad en cambio, muestra estabilidad a lo largo de los años ( $\beta$ n.s., $\left.\mathrm{R}^{2}=0,10 ; \mathrm{F}_{1,27}=3,1, \mathrm{p}=0,09\right)$ (Figura 4). Sin cambios debidos al corte de la cadena de transmisión $\left(\mathrm{F}_{1,25}=1,96, \mathrm{p}=0,17\right)$ ni a la mejoría diagnóstica $\left(\mathrm{F}_{1,25}=3,26, \mathrm{p}=0,08\right)$. La tasa de mortalidad promedio fue $0,36 \pm 0,55$ por 100 mil habitantes; con un $\mathrm{IC}_{0,95}[0,34 ; 0,38]$.

La serie más larga en la que se pudieron obtener datos confiables de infestación domiciliaria (\%) corresponde a

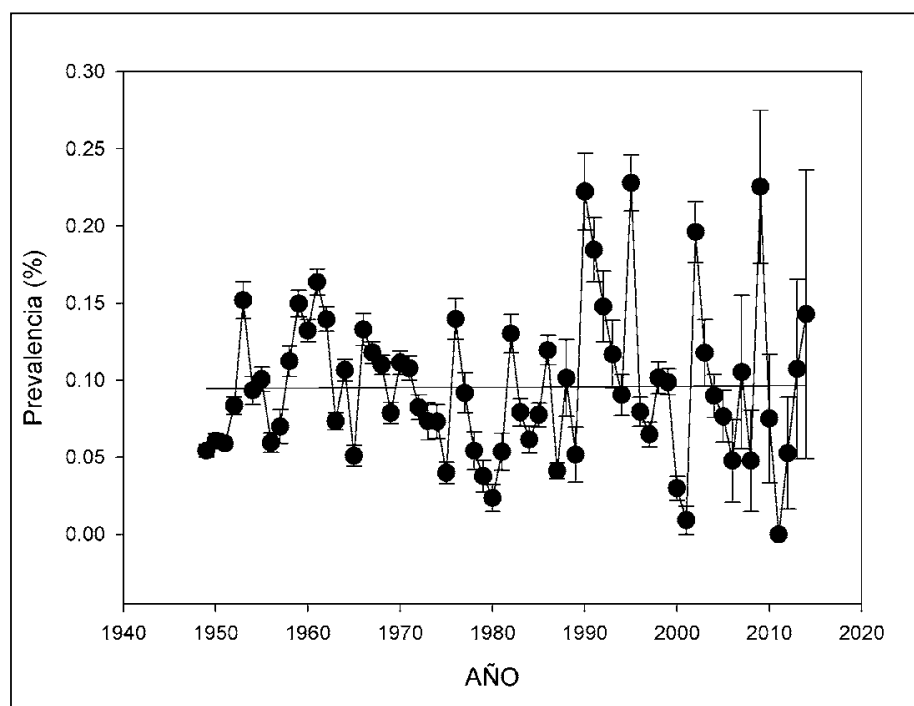

Figura 1. Prevalencia de la enfermedad de Chagas en zonas rurales hiperendémicas 1949-2014, basadas en xenodiagnóstico. La línea muestra la recta de regresión como referencia.

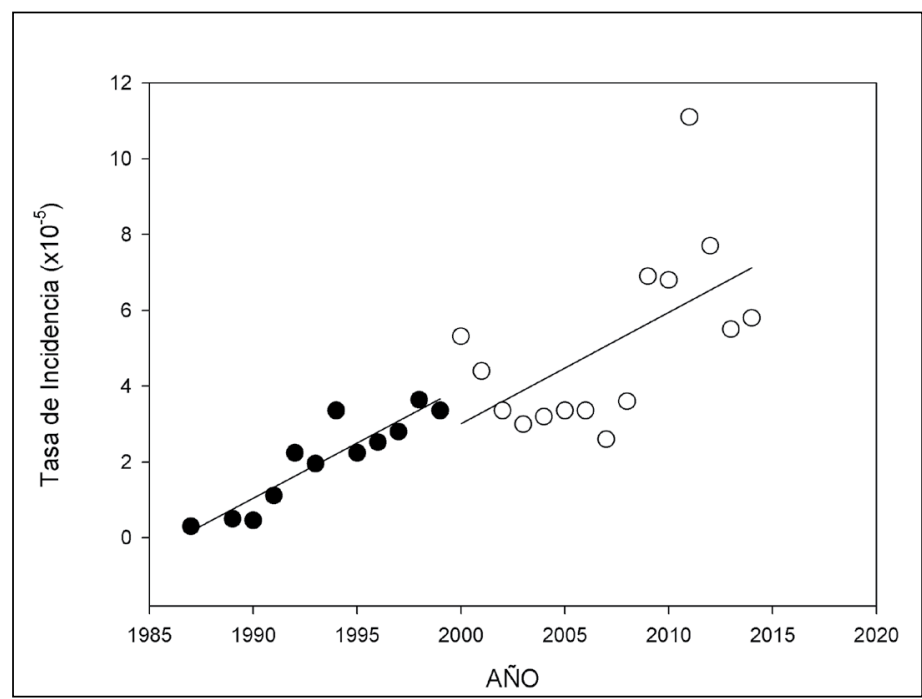

Figura 3. Variación de la tasa de incidencia de enfermedad de Chagas en Chile desde 1987 a 2014, basada en datos del MINSAL ${ }^{3}$. Los círculos negros corresponden al período pre-corte y los círculos blancos post-corte de la cadena de transmisión vectorial. Las líneas representan las rectas de regresión como referencia.

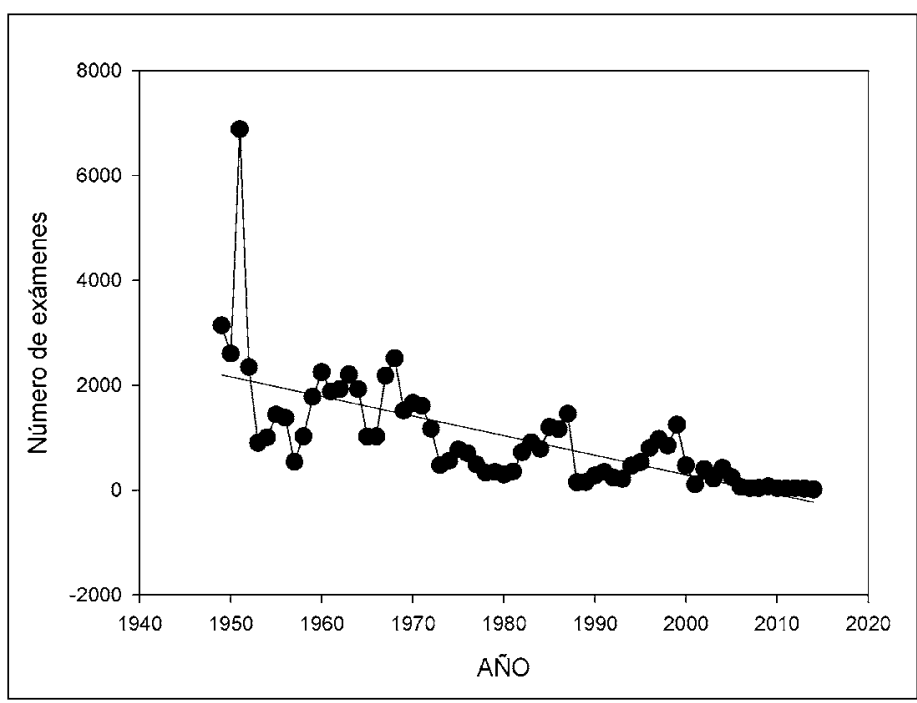

Figura 2. Variación del esfuerzo diagnóstico (número de exámenes de xenodiagnóstico) desde 1949-2014 en el laboratorio de Parasitología de la Facultad de Medicina, Universidad de Chile. La línea muestra la recta de regresión como referencia.

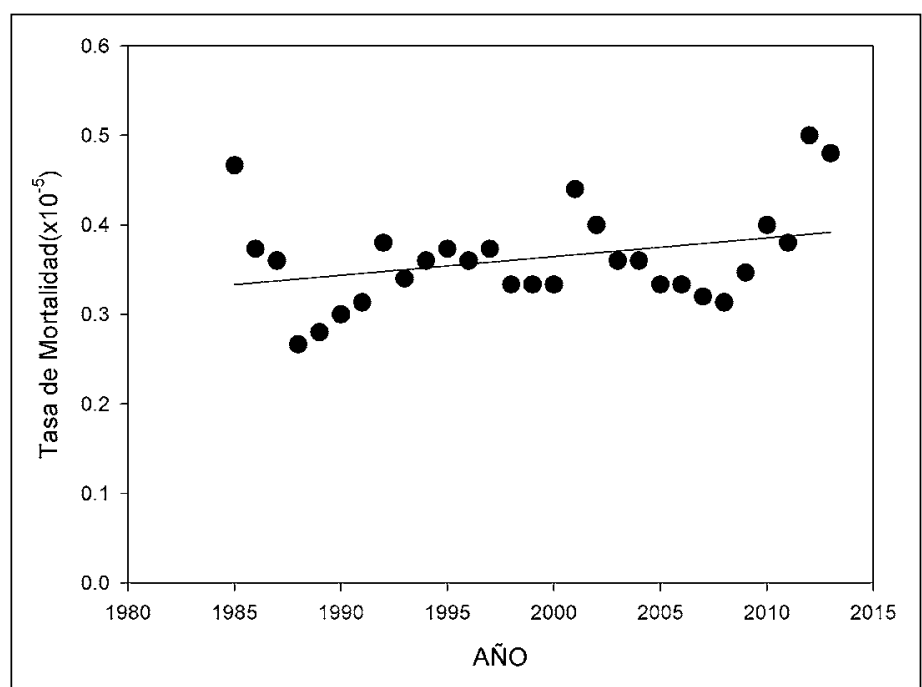

Figura 4. Variación de la tasa de mortalidad de enfermedad de Chagas en Chile desde 1985 a 2013, basada en datos del MINSAL ${ }^{3}$. Las líneas representan la recta de regresión como referencia. 


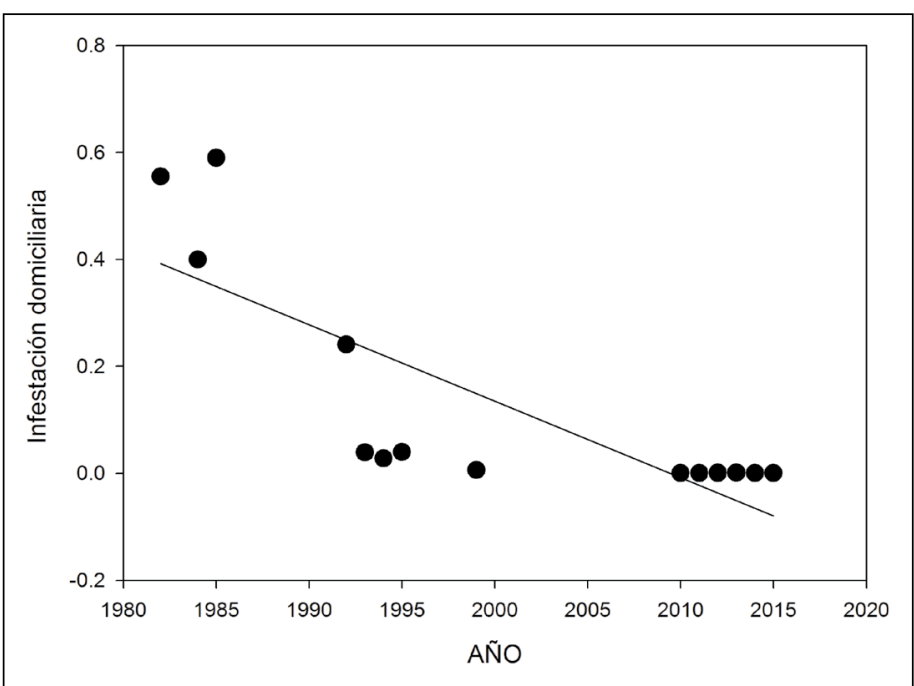

Figura 5. Evolución de la proporción de infestación domiciliaria en la Región de Atacama desde 1982 a 2015 reconstruida a partir de información de la literatura ${ }^{54,55,56}$ y datos ministeriales ${ }^{3}$. La línea representa la recta de regresión como referencia.

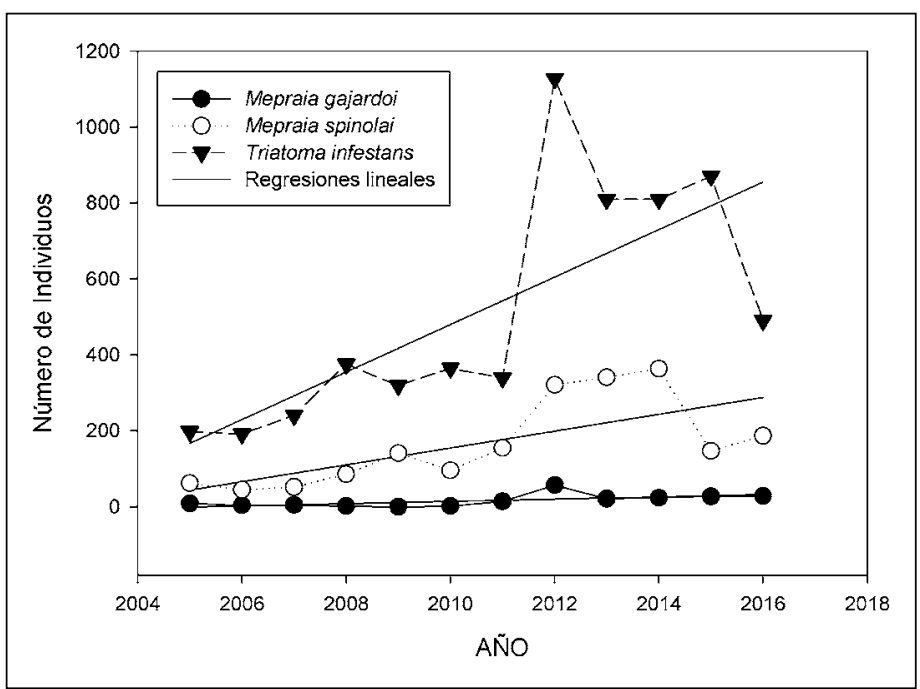

Figura 7. Variación temporal del número de individuos referidos al Instituto de Salud Pública desde 2005 a 2016 para las especies Triatoma infestans, Mepraia spinolai y Mepraia gajardoi. Las líneas representan las rectas de regresión como referencia.

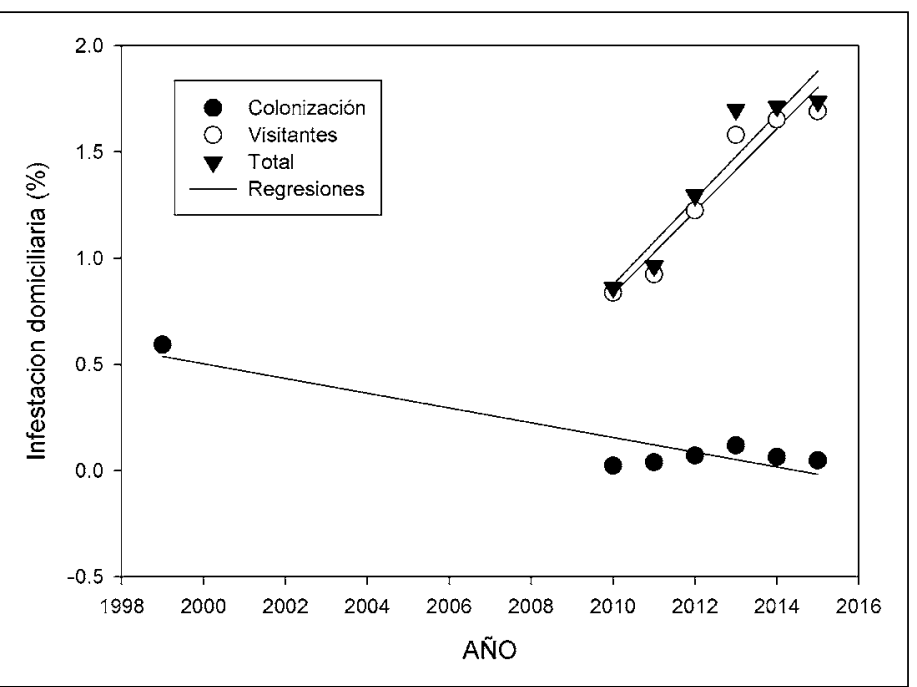

Figura 6. Evolución de la infestación domiciliaria (\%) incluyendo colonias residentes, intromisiones y el total, desde 1999 a 2015, basada en datos del MINSAL ${ }^{3}$. Las líneas representan las rectas de regresión como referencia.

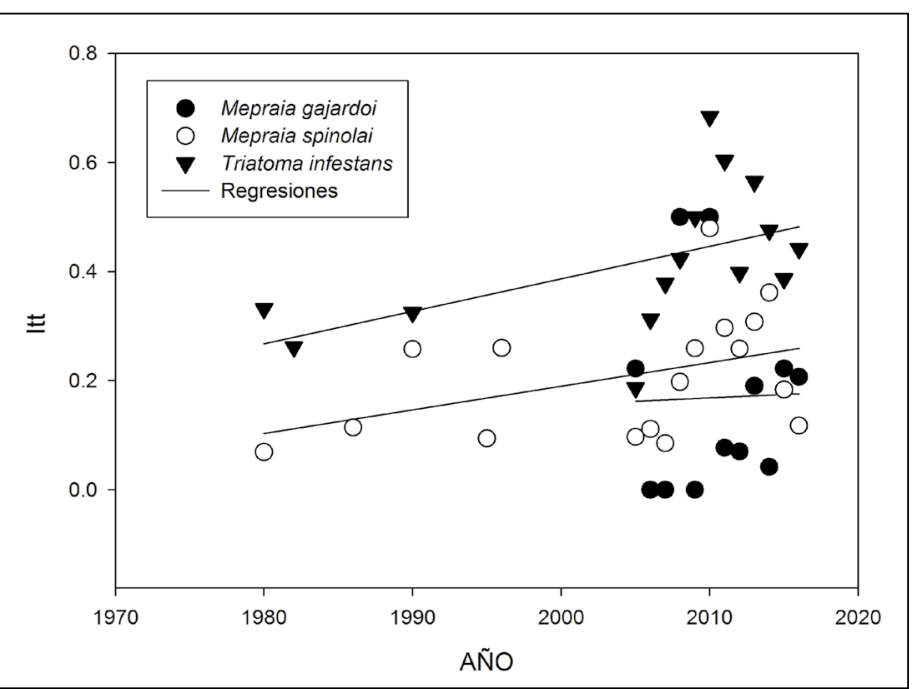

Figura 8. Variación temporal del índice tripano-tratomino (itt: proporción de individuos infectados) en los individuos referidos al Instituto de Salud Pública desde 2005 a 2016 para las especies Triatoma infestans, Mepraia spinolai y Mepraia gajardoi. Los puntos previos al 2005 corresponden a puntos tomados de la literatura ${ }^{7,9-11,22 \text {, }}$ 34-36,38,43,55. Las líneas representan las rectas de regresión como referencia. la serie de Atacama-III ${ }^{\circ}$ Región, en la que se pudieron obtener datos en años saltados desde 1982 a 2015. Esta variable muestra un claro descenso a lo largo de los años $\left(\beta=-0,014, R^{2}=0,64, F_{1,12}=21,0, p=0,0006\right)$ (Figura 5). Las series oficiales del ministerio muestran el dato de 1999 y datos anuales desde 2010, y divide la información en el porcentaje de casas colonizadas, es decir, con evidencias de reproducción de la colonia; las visitas individuales que corresponden a casas que reciben individuos adultos pero sin colonización y el total (la suma de ambos) (Figura 6).
La infestación domiciliaria ha decrecido claramente en este lapso $\left(\beta=-0,000348, R^{2}=0,82 ; F_{1,5}=27,46\right.$, $\mathrm{p}=0,003)$, pero la proporción de casas con visitantes individuales ha incrementado $\left(\beta=0,002, R^{2}=0,91\right.$; $\left.\mathrm{F}_{1,4}=50,64, \mathrm{p}=0,002\right)$ al igual que el total $(\beta=0,002$, $\left.\mathrm{R}^{2}=0,87 ; \mathrm{F}_{1,4}=34,05, \mathrm{p}=0,004\right)$.

Entre 2005 y 2016 se han recibido en el ISP 8.331 vinchucas, de las cuales $73,7 \%$ corresponden $T$. infestans, $24,0 \%$ a M. spinolai y $2,3 \%$ a M. gajardoi. El número de individuos recibidos de las tres especies muestra un 
incremento en estos años $\left(\beta=63,0, R^{2}=0,48 ; F_{1,10}=11,0\right.$, $\mathrm{p}=0,008$ para $T$. infestans, $\beta=22,2, \mathrm{R}^{2}=0,43 ; \mathrm{F}_{1,10}=9,45$, $\mathrm{p}=0,01$ para $M$. spinolai y $\beta=2,93, \mathrm{R}^{2}=0,41 ; \mathrm{F}_{1,10}=$ $6,82, \mathrm{p}=0,025$ para M. gajardoi) (Figura 7). Los índices tripano-triatominos (\% de infección por T. cruzi) de $M$. gajardoi se mantienen claramente estables en el tiempo, aunque basados en un pequeño número de individuos $\left(\beta=0,001, R^{2}=0,006 ; F_{1,10}=0,007, p=0,94\right)$. Los índices de $M$. spinolai y $T$. infestans aunque muestran una leve tendencia positiva, ésta no alcanza significación $\left(\beta=0,011, \mathrm{R}^{2}=0,02 ; \mathrm{F}_{1,10}=1,24, \mathrm{p}=0.29 ; \beta=0,016\right.$, $\mathrm{R}^{2}=0,18 ; \mathrm{F}_{1,10}=2,19, \mathrm{p}=0,17$, respectivamente) (Figura 8). Si para estas dos últimas especies incorporamos reportes previos, de los cuales se tiene información confiable, obtenemos el mismo resultado para $M$. spinolai $\left(\beta=0,004, R^{2}=0,17 ; F_{1,15}=3,02, p=0,10\right)$ y una leve tendencia positiva para $T$. infestans $\left(\beta=0,22, \mathrm{R}^{2}=0,18\right.$; $\left.\mathrm{F}_{1,13}=4,88, \mathrm{p}=0,045\right)$. Los índices promedio fueron: $M$. gajardoi $16,92 \pm 17,77 \%$ con un $\mathrm{IC}_{0,95}[5,32 ; 28,52] ; M$. spinolai $20,88 \pm 11,51 \%$ con un $\mathrm{IC}_{0,95}[15,41 ; 26,35]$ y T. infestans $41,83 \pm 13,23 \%$ con un $\mathrm{IC}_{0,95}[35,13 ; 48,53]$.

\section{Discusión}

Tener una estimación precisa de la enfermedad de Chagas es una tarea difícil ya que depende no sólo de las características del muestreo como el tipo (aleatorio, estratificado, etc.), tamaño de muestra y sesgo, sino también del método de tamizaje utilizado, que en el caso de la enfermedad de Chagas puede ser desde xenodiagnóstico, o ELISA, a inmunofluorescencia y western blot ${ }^{2,3}$. Esto probablemente explica las diferencias en las estimaciones de prevalencia publicadas en la década de los 80/90's y datos actuales. Por ejemplo, entre 1937 y 1980, se reportaban prevalencias promedio en Chile de $16,7 \%{ }^{7}$ con impresionantes valores de $28,5 \%$ para la $\mathrm{I}^{\circ}$ Región, $19,7 \%$ para la $\mathrm{II}^{\circ}, 26,0 \%$ para la $\mathrm{III}^{\circ}, 43,6 \%$ para $\mathrm{la} \mathrm{IV}^{\circ}, 28,1 \%$ para la RM y $23,8 \%$ para la $\mathrm{VI}^{\circ}$ Región. Posteriormente, para 1982-1985, los valores reportados eran menores a excepción de la $\mathrm{VI}^{\circ}$ Región (que tenía un valor excepcionalmente alto), conservando un promedio de $16,9 \%{ }^{33}$. Se puede afirmar con cierta seguridad que estos valores corresponden a valores extremos de personas seleccionadas de regiones rurales hiperendémicas y que no eran extrapolables a la población general en estas regiones. Algo de esto queda en evidencia al comparar estos valores con la información basada en 26.601 donantes de sangre entre 1962 y 1988 que reportaba una prevalencia promedio de 3,7\% distribuida: $I^{\circ}$ Región: 2,8\%; II ${ }^{\circ}$ Región: 4,7\%; III ${ }^{\circ}$ Región: 7,1\%; IV ${ }^{\circ}$ Región: 7,3\%; V ${ }^{\circ}$ Región: 3,1\%; RM: $2,1 \%$, VI ${ }^{\circ}$ Región: $1,6 \%{ }^{11,38}$. Schenone y cols. (1995), notando el sesgo de la información, ya hacen una diferencia entre población rural y urbana muestreada entre
1982 y 1989. Así, mientras la población rural tenía una prevalencia de $16,7 \%$ distribuida: $\mathrm{I}^{\circ}$ Región: 12,5 ; II $^{\circ}$ Región: 10,0\%; III ${ }^{\circ}$ Región: 27,2\%; IV ${ }^{\circ}$ Región: 24,7\%; $\mathrm{V}^{\circ}$ Región: 18,5\%; RM: 11,7\%, $\mathrm{VI}^{\circ}$ Región:7,0\%, la población urbana mostraba una prevalencia promedio de 1,9\% distribuida: $I^{\circ}$ Región: 1,4\%; II $^{\circ}$ Región: 2,2\%; III ${ }^{\circ}$ Región: 3,9\%; IV ${ }^{\circ}$ Región: 3,5\%; $V^{\circ}$ Región: 2,1\%; RM: $1,1 \%$, VI ${ }^{\circ}$ Región: $0,8 \%$. Los valores que reportó la ENS 2009/2010 basados en 4.650 voluntarios mediante ELISA IgG muestran un promedio de $0,7 \%$ de prevalencia promedio, con $1,5 \%$ en población rural y $0,6 \%$ en población urbana, distribuida así: $\mathrm{XV}^{\circ}$ Región: 1,5\%; $I^{\circ}$ Región: 1,3\%; II ${ }^{\circ}$ Región: 1,7\%; III ${ }^{\circ}$ Región: 4,7\%; IV $^{\circ}$ Región: $1,8 \%$; $V^{\circ}$ Región: 0,9\%; RM: 0,7\%, VI ${ }^{\circ}$ Región: 0,7\%; VII ${ }^{\circ}$ Región: 0,2\% y XII ${ }^{\circ}$ Región: 0,3\% (ENS 2009/2010). Estos valores son sorprendentemente parecidos a los reportados para población urbana $82 / 89^{38}$. En este sentido, de no existir sesgos en la ENS, que pueden producirse por ser población voluntaria, esta muestra una evolución de las prevalencias hacia los valores propios de una población urbanizada.

El valor de prevalencia de 9,35\% de este estudio arrastra los sesgos propios de la larga época considerada, gran parte comprendiendo una época cuando las muestras se tomaban a "pulso" optimizando los pocos recursos disponibles estudiando las zonas rurales hiperendémicas; por lo que debe considerarse como una cota superior. Se encuentra en el rango entre 8 y $12 \%$ reportado por Apt y Reyes en $1986^{9}$, también basados en xenodiagnósticos, lo interesante, más que el valor, es que éste no presenta ninguna variación en los 65 años de estudio, independiente de los cambios en los sistemas de salud ni del corte de la cadena de transmisión y tampoco muestra relación con el esfuerzo de muestreo, siendo órdenes de magnitud menor hoy día. Esto último se explica principalmente por la baja en el interés en el estudio de esta patología debido a disminución de la percepción de riesgo y pérdida de la memoria histórica ${ }^{3}$. En este sentido, es interesante notar que la ENS 2009/2010 32 reveló una prevalencia de infección por VIH/SIDA de $0,5 \%$, menor que la de la enfermedad de Chagas.

La evolución de la tasa de mortalidad en Chile tampoco muestra variación evidente, aproximadamente 0,36 por 100 mil habitantes, lo que es completamente consistente con la nula variación de la prevalencia de nuestra muestra. El valor, aunque es un dato duro refrendado en certificados de defunción, probablemente se encuentra subestimado por el subdiagnóstico de esta patología que queda evidenciado por su alta prevalencia. $\mathrm{Si}$, basados en la ENS suponemos una prevalencia de $0,7 \%$, en la RM habría al menos 51.000 pacientes con esta enfermedad.

La incidencia presenta un ascenso progresivo que ocurre desde 1985 en adelante, sin un cambio de pendiente atribuible al corte en la cadena de transmisión vectorial. 
Por otra parte, tampoco se aprecia un efecto notable atribuible a la mejora en la pesquisa. Por ejemplo, el Decreto Supremo 158 de 2004 declara a la enfermedad de Chagas como una enfermedad de declaración obligatoria lo que no se refleja en la curva de tendencia, posteriormente en 2008 la circular 4 instruye a los bancos de sangre a pesquisar la presencia de T. cruzi, en el ordinario 1.839 de 2009 se estipula la política nacional de servicios de la sangre y en 2011 en la circular B51, número 17 se estipula la vigilancia de la enfermedad de Chagas y se establecen formas de registrar e informar a la autoridad sanitaria ${ }^{3}$. Lo único demostrable es que con posterioridad al 2009 la incidencia tiene una relativa estabilización con una pendiente no significativa. Un mayor tamizaje no es suficiente para explicar un ascenso progresivo de la incidencia ya que una mayor cobertura, si bien implica aumentar la detección de casos, también implica un aumento en la población muestreada, es decir, ascensos en el numerador pero también en el denominador de la incidencia. Una explicación para esta dinámica temporal es que el incremento progresivo no fuera explicado por un hito particular sino por una mejora progresiva en la notificación atribuible a una mejor preparación del personal y a un mejor sistema de notificación. Otra posibilidad que no se puede descartar es que este aumento sea real, y en este caso debería repercutir a la larga en las tasas de mortalidad, hecho que aún no es evidente. De persistir la relativa estabilidad detectada desde 2009 estaría indicando que estaría llegando a una estimación adecuada de equilibrio endémico, como predicen los modelos ${ }^{31,39}$.

La infestación domiciliaria muestra un claro descenso, el que es evidente tanto en la serie de Atacama que se pudo reconstruir, como en los datos reportados por el Ministerio de Salud ${ }^{3}$. Sin embargo, llama la atención el aumento en la intromisión de ejemplares adultos provenientes de presuntos focos silvestres. Este resulta también consistente con el aumento que muestran los ejemplares referidos al ISP en las tres especies, M. gajardoi, $M$. spinolai y $T$. infestans. También es destacable que a pesar del referido corte de la cadena de transmisión, 73,7\% de los ejemplares recibidos son de la especie $T$. infestans. Una explicación es que $T$. infestans una vez eliminada de la vivienda humana, ha establecido focos silvestres cuya magnitud no ha sido completamente establecida. Desde la primera descripción de un foco silvestre en Chile en $2003^{34}$ en Calera de Tango y Til-Til, los nuevos focos en Sahondé, Putaendo ( $\mathrm{V}^{\circ}$ Región $)^{35}$ hasta los reportes de focos silvestres en Valparaíso ( $V^{\circ}$ Región) 2009, Atacama 2014 y Coquimbo $2015^{3}$.

Los índices tripano-triatominos son elevados y muestran estabilidad con una tendencia mínimamente ascendente en el caso de T. infestans. Aunque previamente se ha propuesto una escasa participación de los vectores silvestres en la enfermedad de Chagas, basados en una me- nor densidad, un supuesto bajo índice tripano-tratomino y la presencia de $T$. cruzi zimodema Z1 (TCI) con escasa participación en el Chagas humano ${ }^{19,21}$. Sin embargo, esta especie se puede encontrar en altas densidades ${ }^{39-42}$, incluso en colonias mixtas con $T$. infestans y se ha encontrado en el domicilio humano ${ }^{43-45}$. Así, por ejemplo, Schenone y cols. $(1995)^{44}$, reportaron la presencia de 288 ejemplares en 50 viviendas rurales en las regiones $\mathrm{III}^{\circ}, \mathrm{IV}^{\circ}, \mathrm{V}^{\circ} \mathrm{y}$ RM. Desde esta perspectiva, M. spinolai es una especie potencialmente peligrosa, especialmente en las zonas donde se produce el contacto habitual con el hombre como ocurre en las zonas de canteras y en algunas áreas de los alrededores de Santiago donde actualmente se está urbanizando, tales como Colina, Lampa y Til-Til. Además, desde la perspectiva de sus fuentes de alimentación, $M$. spinolai se encuentra en fase de domicialización ${ }^{46}$, con una participación de 7,4\% de sangre humana en su dieta ${ }^{47,48}$. Por otra parte, los estudios actuales muestran una gran susceptibilidad de M. spinolai a diferentes cepas de $T$. cruzi $^{49-52}$ y M. spinolai presenta las cepas TCIIb (Zimodema Z2 $)^{18}$. Así, las premisas que antes permitían proponer que los vectores silvestres tenían escasa participación en la enfermedad de Chagas, no son hoy verdaderas.

Los índices tripano-triatominos en T. infestans permanente elevados son especialmente preocupantes, pues lo esperable era que una vez eliminado de las habitaciones humanas estos valores descendieran. Así, por ejemplo en el caso de Uruguay, los índices se redujeron drásticamente a consecuencia de las acciones de control vectorial ${ }^{53}$. Una explicación para la falta de un descenso en estos índices en Chile podría ser que a pesar del cambio en la dieta que implica la eliminación del ambiente humano, $T$. infestans puede encontrar roedores y otras especies con altos índices de infección que hacen que persistan altos índices tripano triatominos. Esto puede ser efectivamente así, ya que existe una gran diversidad de mamíferos silvestres, domésticos y peridomésticos infectados, algunos llegando a niveles de infección superiores a 10\% $\%^{7,9-11}$.

Finalmente, los números analizados de la enfermedad de Chagas, nos muestran un panorama preocupante en el cual aunque hay evidencias de un descenso en las tasas de infestación domiciliaria, ésta no repercute aún sobre los indicadores de enfermedad en el hombre como prevalencia, incidencia y mortalidad. Más aún, la situación de los vectores es también preocupante ya que a pesar de la disminución de la infestación domiciliaria, hay vectores silvestres que podrían cobrar importancia, se reportan más focos silvestres de $T$. infestans, aumentan las intromisiones al domicilio y, por otra parte, los vectores muestran altos índices de infección. Son amenazas evidentes la pérdida de la percepción del riesgo y la pérdida de la memoria histórica (MINSAL 2016). Mientras la preocupación por esta enfermedad parece ir decreciendo con menores esfuerzos diagnósticos y menor enseñanza 
a nivel superior, por otra parte, los números muestran que el problema si es que no está aumentando, al menos mantiene su descuidada magnitud histórica.

Agradecimientos. Los autores agradecen al Dr. Alonso Parra por aportar la información del reporte del Ministerio de Salud julio de 2016 y por la agradable discusión del tema.

\section{Resumen}

Introducción: La enfermedad de Chagas sigue siendo altamente prevalente en Chile, especialmente entre las regiones de Arica y Parinacota y de Coquimbo. Desde 1999 se considera que en Chile se encuentra interrumpida la transmisión vectorial. Bajo esta premisa, la dinámica epidemiológica se debiera estar modificando. Objetivo: Analizar la evolución temporal de la enfermedad de Chagas en Chile Material y Métodos: Analizamos la evolución de la prevalencia de la enfermedad de Chagas a través del análisis de resultados de 64.995 xenodiagnósticos realizados en el laboratorio de Parasitología de la Facultad de Medicina de la Universidad de Chile, entre 1949 y 2014. Se estudió la evolución de las mortalidades e incidencias disponibles en las bases de datos del Ministerio de Salud en los períodos en que fue posible.
Se analizaron las tasas de infestación domiciliaria y el número de insectos vectores enviados al Instituto de Salud Pública y sus índices tripano-tratominos. Resultados: La prevalencia de la enfermedad de Chagas en habitantes de zonas de riesgo se mantuvo estable en este período, al igual que la mortalidad. La tasa de incidencia muestra un incremento progresivo con tendencia a la estabilización. Se encontró un significativo decrecimiento del esfuerzo de muestreo, decayendo dos órdenes de magnitud, especialmente desde 2000. El aumento progresivo de la morbilidad no tiene clara relación con el corte de la cadena vectorial ni con el mayor esfuerzo diagnóstico ocurrido en 2009, ya que era evidente desde antes. Mientras que la infestación domiciliaria disminuye, han aumentado los reportes de intromisión de individuos solitarios y los focos silvestres de T. infestans. Los índices tripano triatominos se mantienen con valores altos en todas las especies vectores. Discusión: Este estudio muestra una situación preocupante, ya que mientras por una parte se destaca el corte de la transmisión vectorial y mejora en los sistemas de pesquisa, la preocupación por esta enfermedad parece ir decreciendo con menores esfuerzos diagnósticos y menor enseñanza a nivel superior, y por otra parte los números muestran que el problema si es que no está aumentando, al menos mantiene su descuidada magnitud histórica.

\section{Referencias bibliográficas}

1.- Hotez P J, Bottazzi M E, Franco-Paredes C, Ault S, Periago M R. The neglected tropical diseases of Latin America and the Caribbean: a review of disease burden and distribution and a roadmap for control and elimination. PLoS Negl Trop Dis 2008; 2: e300. doi:10.1371/ journal.pntd.0000300.

2.- MINSAL, 2014. Norma general técnica. Control y prevención nacional de la enfermedad de Chagas. Febrero. Ministerio de Salud de Chile.

3.- MINSAL, 2016. Informe Programa Nacional Integral de Enfermedad de Chagas, Julio. Disponible en http://epi.minsal. cl/chagas-materiales-relacionados/ (Consultado en septiembre de 2016).

4.- Hotez P J, Dumonteil E, Woc-Colburn L, Serpa J A, Bezek S, Edwards M S, et al. Chagas disease: "The New HIV/AIDS of the Americas". PLoS Negl Trop Dis 2012; 6 (5): e1498. doi:10.1371/journal.pntd.0001498

5.- Cucunubá Z M, Okuwoga $\mathrm{O}$, Basáñez M G, Nouvellet P. Increased mortality attributed to Chagas disease: a systematic review and meta-analysis. Parasite \& Vectors 2016; 9: 42. DOI 10.1186/s13071-016-1315-x

6.- Fuentes R, Maturana M, De la Cruz R. Eficacia de nifurtimox para el tratamiento de pacientes con enfermedad de Chagas crónico. Rev Chilena Infectol 2012; 29 (1), 82-6.

7.- Schenone H, Villarroel F, Rojas A, Alfaro E. Factores biológicos y ecológicos en la epidemiología de la enfermedad de Chagas en Chile. Bol Chil Parasitol 1980; 35: 42-54.

8.- Schenone H, Rojas A. Algunos datos y observaciones pragmáticas en relación a la epidemiología de la enfermedad de Chagas. Bol Chil Parasitol 1989; 44: 66-86.

9.- Apt W, Reyes H. Aspectos epidemiológicos de la enfermedad de Chagas en Chile. I: Distribución geográfica, índices de infección en vectores y en humanos. Parasitol Día 1986; 10: 84-101.

10.- Apt W, Reyes H. Aspectos epidemiológicos de la enfermedad de Chagas en Chile. II: Infección en animales, algunas características especiales del problema, el control. Parasitol Día 1986; 10: 129-33.

11.- Apt W, Reyes H, Algunos aspectos de la enfermedad de Chagas en Latinoamérica. Parasitol Día 1990; 14: 23-40.

12.- Canals M, Cattan P E, Valderas J, Solís R. Biología de poblaciones de Triatoma infestans: Fluctuaciones de la mortalidad y fertilidad. Rev Med Chile 1991a; 119 (9): 79-983.

13.- Canals M, Cattan P E, Valderas J, Solís R.
Efectos poblacionales de fluctuaciones de mortalidad y fecundidad en Triatoma infestans: Simulación mediante matrices de Leslie. Rev Med Chile 1991b; 119 (11): 1239-42.

14.- Canals M, Cattan P E, Ehrenfeld M, Torres P. Poblaciones experimentales de $T$. infestans: efectos de condiciones ambientales variables. Parasitol Día 1992a; 16 (3-4): 72-7.

15.- Ehrenfeld M, Canals M, Cattan P E. Population parameters of Triatoma spinolai under different environmental conditions and densities. J Med Entomol 1998; 35 (5): 740-4.

16.- Carcavallo R U, Galíndez I, Jurberg J, Lent H. 1998. Atlas of Chagas'disease vectors in the Americas. R. U. Carcavallo, I. Galíndez Girón, J. Jurberg, and H. Lent, Eds. Rio de Janeiro, Editora FIOCRUZ.

17.- Botto-Mahan C, Cattan P E, Canals M. Field test of carbon dioxide and conspecifics as baits for Meparia spinolai, wild vector of Chagas disease. Acta Tropica 2002; 82: 377-80.

18.- Botto-Mahan C, Ortiz S, Rozas M, Cattan P E, Solari A. DNA evidence of Trypanosoma cruzi in the Chilean wild vector Mepraia spinolai (Hemiptera: Reduviidae). Mem Inst Oswaldo Cruz 2005a; 100: 237-9.

19.- Canals M, Cattan P E, Ehrenfeld M. Algunas 
estimaciones numéricas de la importancia epidemiológica de los vectores de la enfermedad de Chagas en Chile. Parasitol Día 1993; 17: 79-86.

20.- Canals M, Ehrenfeld M, Solís R, Cruzat L, Pinochet A, Tapia C, et al. Biología comparada de Mepraia Spinolai en condiciones de laboratorio y terreno: cinco años de estudio. Parasitol Día 1998; 22: 72-8.

21.- Canals M, Bustamante R O, Ehrenfeld M, Cattan P E. Assesing The impact of insect vectors on animal populations. Acta Biotheoretica 1999; 46: 337-45.

22.- Órdenes H, Ehrenfeld M, Cattan P E, Canals M. Infección tripano-triatomina de Triatoma spinolai en una zona de riesgo epidemiológico. Rev Med Chile 1996; 124: 1053-7.

23.- Da Silva N N, Clausell D T, Nolibos H, de Mello A L, Ossanai J, et al. Surto epidêmico da doença de Chagas com provável contaminação oral. Rev Inst Med Trop Sao Paulo 1968; 10 (5): 265-76.

24.- Nóbrega A A, García M H, Tatto E, Obara M T, Costa E, Sobel J. Oral transmission of Chagas disease by consumption of acai palm fruit, Brazil. Emerg. Infect. Dis 2009; 15(4): 653-5.

25.- Shikanai-Yasuda M A, Marcondes C B, Guedes L A. Possible oral trasmission of acute Chagas'disease in Brazil. Rev Inst Med Trop Sao Paulo 1991; 33: 351-7.

26.- Apt W, Heitmann G, Jercic M I, Jofré L, Muñoz C del V P, Noemí I, et al. Guías clínicas de la enfermedad de Chagas. Parte I. Introducción y epidemiología. Rev Chilena Infectol 2008; 25 (3): 189-93.

27.- Tilleria J, Tibayrenc M. 2010. American trypanosomiasis. Chagas disease. One hundred years of research. Amsterdam, Elsevier.

28.- Rassi Jr A, Rassi A, Marcondes de Rezende J. American Trypanosomiasis (Chagas Disease). Infect Dis Clin North Am. 2012; 26: 275-91.

29.- Raimundo S M, Massad E, Yang H M. Modelling congenital transmission of Chagas, disease. Biosystems 2010; 99: 215-22.

30.- Massad E. The elimination of Chagas disease from Brazil. Epidemiol. Infect 2008; 136: 1153-64.

31.- Canals M, Cáceres D, Alvarado S, Canals A, Cattan P E. Modeling the Chagas disease: from the vectorial to congenital transmission. Biosystems 2006; (submitted).

32.- Encuesta Nacional de Salud ENS Chile 2009-2010. Ministerio de Salud.

33.- Schenone H, Christensen H A, De Vásquez AM. Fuentes de alimentación de triatomas domésticos y su implicancia epidemiológica en relación a la enfermedad de Chagas en áreas rurales de siete regiones de Chile. Bol Chil Parasitol 1985; 40: 34-8.

34.- Bacigalupo A, Segura J A, García A, Hidalgo J,
Galuppo S, Cattan P E. Primer hallazgo de vectores de la enfermedad de Chagas asociados a matorrales silvestres en la Región Metropolitana, Chile. Rev Med Chile 2006; 134: $1230-6$

35.- Bacigalupo A, Torres-Pérez F, Segovia V, García A, Correa J P, Moreno L, et al. Sylvatic foci of the Chagas disease vector Triatoma infestans in Chile: description of a new focus and challenges for control programs. Mem. Inst. Oswaldo Cruz 2010; 105 (5): 633-41.

36.- Bacigalupo A, Correa J P, García A, Cattan P E. Focos silvestres de Triatoma infestans en Latinoamérica: análisis y perspectivas para Chile. Parasitol Latinoam 2015; 64 (3): 33-42.

37.- Anuarios de Enfermedades de Enfermedades de Notificación Obligatoria ENO. 1985, 1987, 1989, 1990,1991. Ministerio de Salud.

38.- Schenone H, Contreras MC, Salinas P, Sandoval L, Rojas A, Villarroel F. Epidemiología de la enfermedad de Chagas en Chile. Frecuencia de infección humana por Trypanosoma cruzi por grupos de edad y regiones. Bol Chil Parasitol 1995; 50: 84-6.

39.- Canals M, Cattan P E. Dinámica de transmisión de la infección de la infección chagásica en Chile: Modelo y simulación. Rev Med Chile 1992; 120 (12): 1359-65.

40.- Botto-Mahan C, Campos R, Acuña-Retamar M, Coronado X, Cattan P E, et al. Temporal variation of Trypanosoma cruzi infection in native mammals in Chile. Vector-borne Zoonotic Dis 2010; 10: 317-9.

41.- Botto-Mahan C, Correa J P, Bacigalupo A, Campos-Soto R, Cattan P E, Solari A. Ecología de los triatominos silvestres endémicos de Chile. Parasitol Latinoam 2015; 64 (3): 17-24.

42.- Cattan P E, Pinochet C, Botto-Mahan C, Acuña M I, Canals M. Abundance of Mepraia spinolai in a periurban zone of Chile. Mem Inst Oswaldo Cruz 2002; 97 (3): 285-7.

43.- Frías-Lasserre D. A New species and karyotype variation in the bordering distribution of Mepraia spinolai (Porter) and Mepraia gajardoi Frías et al (Hemiptera: Reduviidae: Triatominae) in Chile and its parapatric model of speciation. Neotrop Entomol 2010; 39 (4): $572-83$.

44.- Schenone H, Villarroel F, Rojas A. Presencia de Triatoma spinolai en viviendas humanas. Bol Chil Parasitol 1995; 50 (3-4): 76-80.

45.- Canals M, Cattan P E, Ehrenfeld M. Sobrevivencia de Triatoma spinolai en ambiente habitacional. Parasitol Día 1994; 18 (3): 82-7.

46.- Canals M, Ehrenfeld M, Cattan P E. Situación de Mepraia Spinolai, vector silvestre de la enfermedad de Chagas en Chile, en relación con otros vectores, desde la perspectiva de sus fuentes de alimentación. Rev Med Chile 2000; 128: $1108-12$.

47.- Canals M, Cruzat L, Ehrenfeld M, Molina M C, Ferreira A, Cattan P E. Blood sources of Mepraia spinolai (Hemiptera Reduviidae), wild vector of Chagas's disease in Chile. J Med Entomol 2001; 38 (2): 303-7.

48.- Molina M C, Cattan P E, Canals M, Cruzat L, Aguillon J C, Ferreira A. A simple immunometric assay to asses the feeding habits of Mepraia spinolai, a Tripanosoma cruzi vector. Parasitol Res 2004; 92: 375-9.

49.- Rozas M, Botto-Mahan C, Coronado X, Ortiz S, Cattan P E, Solari A. Co-existence of Trypanosoma cruzi genotypes in wild and peridomestic mammals in Chile. Am J Trop Med Hyg 2007; 77: 647-53.

50.- Rozas M, Botto-Mahan C, Coronado X, Ortiz S, Cattan P, Solari A. Trypanosoma cruzi infection in wild mammals from a chagasic area of Chile. Am J Trop Med Hyg 2005; 73: 517-9.

51.- Campos R, Acuña-Retamar M, Botto-Mahan C, Ortiz S, Cattan P, Solari A. Susceptibility of Mepraia spinolai and Triatoma infestans to different Trypanosoma cruzi strains from naturally infected rodent hosts. Acta Tropica 2007; 104: 25-9.

52.- Botto-Mahan C, Sepúlveda M, Vidal M, AcuñaRetamar M, Ortiz S, Solari A. Trypanosoma cruzi infection in the wild kissing bug Mepraia gajardoi from the Chilean Southern Pacific Ocean coast. Acta Tropica 2008; 105: 166-9.

53.- Salvatella R. Evolución del índice de infección tripanosómica en Tríatoma infestans (KlugJ834) con las acciones de control antivectorial, en dos áreas endémico chagásicas de Uruguay. Rev Med Uruguay 1995; 11 : 105-10.

54.- González J, Contreras M C, Schenone H, Adaos H, Cabezas R. Enfermedad de Chagas Impacto del programa de control de Triatoma infestans en la comuna de Alto del Carmen, provincia de Huasco, III $^{\circ}$ Región, Atacama, Chile. Bol Chil Parasitol 1996; 51 (1-2): 28-30.

55.- Rodríguez J, Bertoglia J, Gordillo N, Mendoza J, Rojas J, Contreras M C, et al. Infestación triatomina domiciliaria e infección por Trypanosoma cruzi en la III $^{\circ}$ Región Atacama, Chile. Bol Chile Parasit 1982; 37: 29-30.

56.- Schenone H, Contreras M C, Borgoño J M, Rojas A, Vollarroel F, Valdés J. Enfermedad de Chagas en Chile. Sectores rurales y urbanos de zona de endemo-enzootia. Relaciones de las condiciones de la vivienda, infestación triatomínea domiciliaria e infección por Trypanosoma cruzi del vector, del humano y de mamíferos domésticos. 1982-1985. Bol Chile Parasit 1985; 40 (3-4): 58-67. 